\title{
Glycosaminoglycans are important mediators of neutrophilic inflammation in vivo
}

Martha Gschwandtner ${ }^{\mathrm{a}}$, Elisabeth Strutzmann", Mauro M Teixeira ${ }^{\mathrm{b}}$, Hans J Anders ${ }^{\mathrm{c}}$, Maria Diedrichs-Möhring ${ }^{\mathrm{d}}$, Tanja Gerlza ${ }^{\mathrm{a}, \mathrm{e}}$, Gerhild Wildner ${ }^{\mathrm{d}}$, Remo C Russo ${ }^{\mathrm{b}}$, Tiziana Adage ${ }^{\mathrm{e}}$, Andreas J Kungl a,e,1

${ }^{a}$ Institute of Pharmaceutical Sciences, Department of Pharmaceutical Chemistry, University of Graz, Schubertstraße 1/1, 8010 Graz, Austria

${ }^{\mathrm{b}}$ Institute of Immunology, Department of Biochemistry and Immunology, Universidade Federal de Minas, Avenida Antonio Carlos, Belo Horizonte 31270-901, Brazil

'Institute of Clinical Biochemistry, Medical Policlinic, Klinikum der Universität München, Schillerstr. 42, 80336 München, Germany

${ }^{\mathrm{d} S e c t i o n}$ of Immunobiology, Department of Ophthalmology, Klinikum der Universität München, Germany. Mathildenstr. 8, 80336 München, Germany

e ProtAffin Biotechnolgie AG, Reininghausstraße 13a, 8020, Graz, Austria

E-mail addresses: martha.gschwandtner@edu.uni-graz.at, elisabeth.strutzmann@gmail.com, mmtex@icb.ufmg.br, Maria.Diedrichs-Moehring@med.uni-muenchen.de, tanja.gerlza@unigraz.at, Gerhild.Wildner@med.uni-muenchen.de, remo@icb.ufmg.br, tadage@hotmail.com, andreas.kungl@uni-graz.at 
${ }^{1}$ Address correspondence to this author at the Institute of Pharmaceutical Sciences, Department of Pharmaceutical Chemistry, University of Graz, Schubertstraße 1/1, 8010 Graz, Austria, Tel: +43 31603805373, E-mail: andreas.kungl@uni-graz.at

\section{ABBREVIATIONS}

BAL, bronchoalveolar lavage; BID, [bis in die] twice a day; CS, chondroitin sulphate, CXCL8, CX-C motif chemokine 8/ Interleukin-8; CXCR1/2; chemokine (C-X-C motif) receptor 1/2; EAU, experimental autoimmune uveitis; ELICO, ELISA-like competition assay; ELR, glutamic acidleucine-arginine; FI, fluorescence intensity; GAG, glycosaminoglycan; GPCR, G protein-coupled receptor; HBSS-/-, Hank’s Balanced Salt Solution (without calcium and magnesium); HMWH, high molecular weight heparin; HS, heparan sulphate; i.t., intratracheally; IPF, idiopathic pulmonary fibrosis; KC, murine CXCL1; LMWH, low molecular weight heparin; MIP-2, murine CXCL2; PA401, dominant negative Interleukin-8 mutant; PBMC, peripheral blood mononuclear cell; PBS, phosphate buffered saline; PDSAg peptide, pathogenic peptide derived from retinal Santigen (S-Ag); s.c., subcutaneously; UPEC, uropathogenic Escherichia coli; UTI, urinary tract infection 


\section{ABSTRACT}

The pro-inflammatory chemokine Interleukin-8 (CXCL8) exerts its function by establishing a chemotactic gradient in infected or damaged tissues to guide neutrophil granulocytes to the site of inflammation via its G protein-coupled receptors (GPCRs) CXCR1 and CXCR2 located on neutrophils. Endothelial glycosaminoglycans (GAGs) have been proposed to support the chemotactic gradient formation and thus the inflammatory response by presenting the chemokine to approaching leukocytes. In this study, we show that neutrophil transmigration in vitro can be reduced by adding soluble GAGs and that this process is specific with respect to the nature of the glycan. To further investigate the GAG influence on neutrophil migration, we have used an engineered CXCL8 mutant protein (termed PA401) which exhibits a much higher affinity towards GAGs and an impaired GPCR activity. This dominant-negative mutant chemokine showed antiinflammatory activity in various animal models of neutrophil-driven inflammation, i.e. in urinary tract infection, bleomycin-induced lung fibrosis, and experimental autoimmune uveitis. In all cases, treatment with PA401 resulted in a strong reduction of transmigrated inflammatory cells which became evident from histology sections and bronchoalveolar lavage. Since our CXCL8based decoy targets GAGs and not GPCRs, our results show for the first time the crucial involvement of this glycan class in CXCL8/neutrophil-mediated inflammation and will thus pave the way to novel approaches of anti-inflammatory treatment.

\section{KEYWORDS}

CXCL8, chemokine, glycosaminoglycan, urinary tract infection, bleomycin model, experimental autoimmune uveitis 


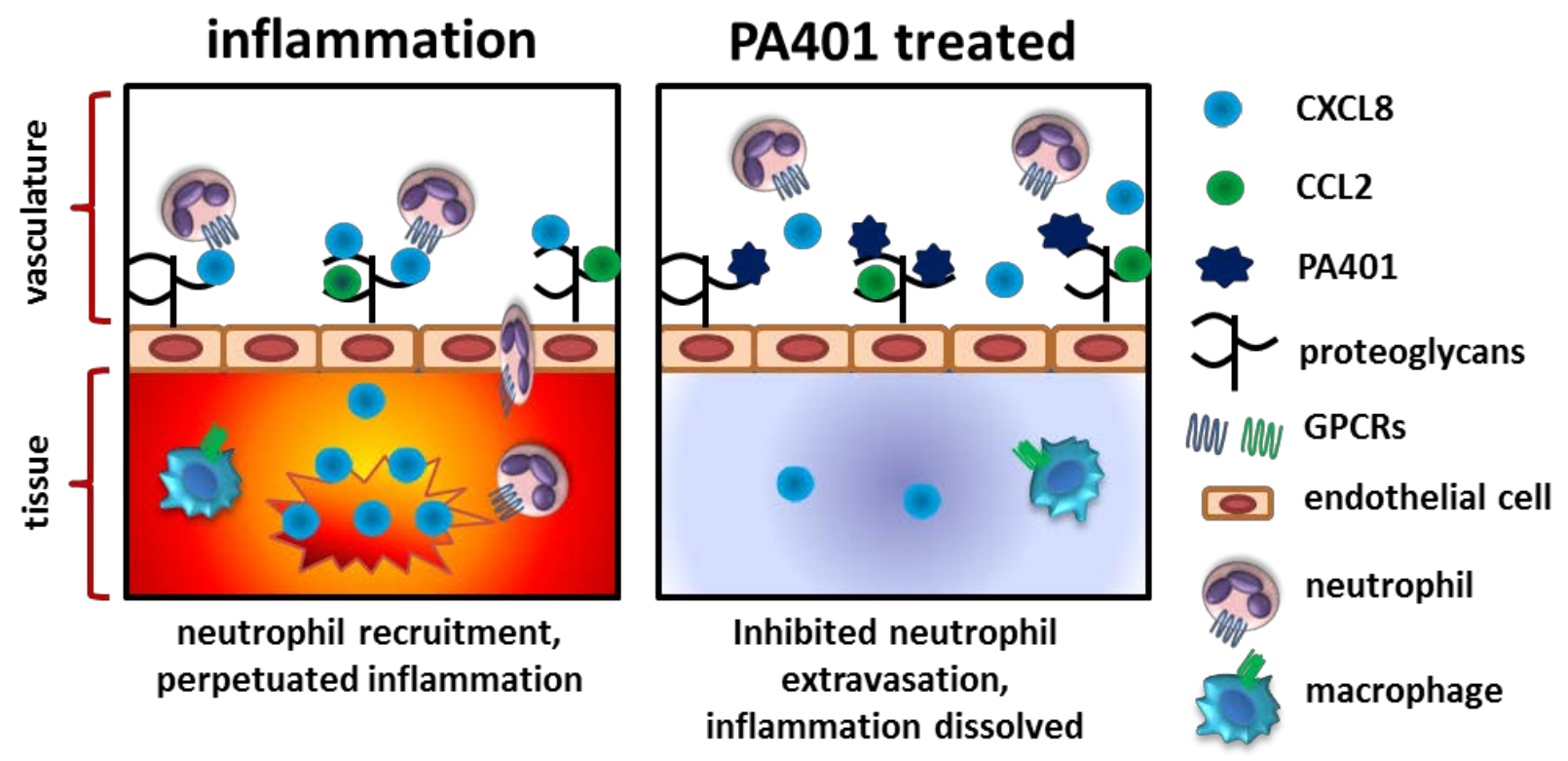

\section{INTRODUCTION}

\subsection{Interleukin-8}

The chemokine Interleukin-8 (IL-8; CXCL8) is a member of the CXC chemokine family and can be classified as pro-inflammatory chemokine due to its secretion at sites of inflammation by cytokine-activated endothelial cells and its chemotactic properties towards several types of inflammatory cells [1]. CXCL8 is involved in several pathologic processes such as lung diseases (chronic obstructive pulmonary disease, cystic fibrosis and asthma), autoimmune diseases (rheumatoid arthritis, psoriasis) and early tissue injury [2, 3] . It exerts its pro-inflammatory effects by triggering neutrophil granulocytes via its specific seven-transmembrane spanning G proteincoupled receptors (GPCRs), CXCR1 and CXCR2, as well as via binding to its proposed coreceptors, the endothelially presented proteoglycans [4]. 


\subsection{Proteoglycans/Glycosaminoglycans}

Proteoglycans, which are located on the cell surface of eukaryotic cells, consist of a core protein on which glycosaminoglycan (GAG)-chains are covalently attached. GAGs, e.g. heparan sulphate (HS), heparin, chondroitin sulphate (CS), are highly sulphated, unbranched, O-linked heteropolysaccharides that are composed of repeating disaccharide units. Their molecular size and their sulphation patterns depend on the type and condition of the cells on which they are expressed [5].

\subsection{Interleukin-8 GAG interaction}

In the course of inflammation chemokines such as CXCL8 are presented by GAGs on the surface of endothelial cells as well as forming a chemotactic gradient in the infected or damaged tissue, which are crucial steps in the recruitment of leukocytes in vivo[6, 7]. Binding of leukocytes to the chemokines presented by the endothelium leads to leukocyte activation, firm adhesion, transmigration across the endothelial barrier and accumulation of leukocytes at the centre of inflammation, where they further promote the immune response [7].

The interaction of CXCL8 and GAGs is driven by strong ionic forces between the sulphate groups of the carbohydrates and the basic residues of the protein. Specificity however is introduced through weak forces like hydrogen bonding and van der Waals forces [7]. The residues of CXCL8 involved in GAG-binding have been identified to include $\mathrm{Arg}^{60}, \mathrm{Lys}^{64}$, $\mathrm{Lys}^{67}$, $\mathrm{Arg}^{68}$ in the Cterminal alpha-helix as well as His ${ }^{18}$ and Lys $^{20}$ located in the proximal loop [8]. The residues of CXCL8 contributing to the binding and activation of CXCR1 and CXCR2 are the N-terminal ELR (glutamic acid-leucine-arginine) motif as well as a hydrophobic pocket in the proximal N-loop comprising of $\mathrm{Ile}^{10}, \mathrm{Tyr}^{13}$, $\mathrm{Phe}^{17}$, $\mathrm{Phe}^{21}$ and $\mathrm{Ile}^{22}[9]$. 


\subsection{Dominant negative engineering of Interleukin-8}

We have recently engineered a dominant-negative CXCL8 mutant, termed PA401, with knockedout GPCR binding and increased GAG binding affinity [10]. This mutant showed good antiinflammatory activity in LPS- and tobacco-induced lung inflammation [11]. Here we have used this CXCL8 mutant to further prove the involvement of GAGs in diverse CXCL8-mediated, neutrophil-related disease models based on the strong, GPCR-independent, anti-inflammatory activity of PA401.

\section{MATERIALS AND METHODS}

\subsection{Chemicals and reagents}

Chemicals were purchased from Sigma-Aldrich (St. Louis, MO), unless stated otherwise. Dominant negative (dn) CXCL8 decoy (PA401) was designed and produced by ProtAffin Biotechnologie AG (Graz, Austria) as described in [10, 12].

\subsection{Transendothelial migration assay}

Membranes of the transwell plates (HTS Transwell-96 Well Plate, PC membrane with $5 \mu \mathrm{M}$ pore size, Corning, USA) were coated with collagen (from rat tail) using a final concentration of $1 \mu \mathrm{g} / 25$ $\mu \mathrm{L}$ collagen in $0.1 \%$ acetic acid per well in the upper compartment and an incubation time of 2 hrs at $37^{\circ} \mathrm{C}, 5 \% \mathrm{CO}_{2}$. After the incubation time, wells were washed with HBSS-/- (100ul/well), air-dried, and cells were seeded and grown in the wells for three days before the assay. EA.hy926 (CRL-1730, ATCC, USA) endothelial cells were trypsinated from their cultivation flask and diluted with full growth medium (consisting of DMEM (Gibco, USA), 10 \% FBS (GE Healthcare, 
USA), 4 mM L-Glutamine (GE Healthcare, USA) and 1\% Pen/Strep 1:100 (GE Healthcare, USA)) to a final concentration of 7000 cells/75 $\mu \mathrm{L} /$ well. Cells were filled into the upper well and $220 \mu \mathrm{L}$ cultivation medium was added to the lower transwell compartment.

On the day of the assay human neutrophils were isolated from whole blood of healthy voluntary donors via density gradient centrifugation using Ficoll paque plus (GE Healthcare, USA) followed by a dextran gradient separation. The suspension cells were labelled using Calcein-AM.

200000 cells were needed per well, so the respective number of cells was harvested by centrifugation and resuspended in 10 mL HBSS-/- (PAA Laboratories GmbH, Linz/Austria). 10 $\mu \mathrm{L}$ of $2 \mathrm{mM}$ Calcein-AM were added to the cells and incubated for $30 \mathrm{~min}$ at $37^{\circ} \mathrm{C}$ and $5 \% \mathrm{CO}_{2}$. After labelling, suspension cells were harvested by centrifugation, washed with $10 \mathrm{~mL}$ HBSS-/-, divided in the number of treatment schemes in different tubes, again harvested by centrifugation, resuspended in the decoy protein/ GAG dilutions and pipetted in the allocated well. $10 \mu \mathrm{g} / \mathrm{mL}$ PA401 ( $=1,3 \mu \mathrm{M}$ for the monomeric protein) and $10 \mu \mathrm{g} / \mathrm{mL}$ GAG concentrations were used for inhibition of chemotaxis, with $10 \mathrm{nM}$ CXCL8 as chemoattractant in the lower compartment.

The transmigration assay was run for two hours at $37^{\circ} \mathrm{C}$ and $5 \% \mathrm{CO}_{2}$. Detection was performed by measuring the fluorescence intensity (FI) of migrated cells in the lower wells at $485 \mathrm{~nm}$ (excitation)/ 535 nm (emission) using the SpectraMax M3 Plate Reader (Molecular Devices, USA).

\subsection{ELICO- chemokine displacement fingerprint}

The ELICO measurements were conducted as described in a recently published procedure [13]. Different types of chemokines, being CCL2, CCL3, CCL5, CCL11, CXCL4, CXCL8, CXCL10, 
CXCL11, CXCL12, were incubated (250 nM) on a HS coated plate and PA401 (100 $\mu$ M to 6 nM) was used as competitor for HS-binding.

\subsection{Animal experiments}

Animal care and handling procedures including providing of food and water ad libitum, 12-hour light and dark cycle and keeping in ventilated cages were performed in accordance with the European guidelines (and Brazilian for the bleomycin model) and all the experiments were conducted under conditions previously approved by the local animal ethics committees.

\subsection{Murine model of urinary tract infection (UTI)}

Female C3H/HeN wild type mice were obtained from Charles River, Germany. Mice were divided in 2 groups of 10 mice each, that received a single intraperitoneal injection of either $1 \mu \mathrm{g}$ of the CXCL8-based decoy (PA401) in $100 \mu \mathrm{L}$ saline or saline only (control group) 10 minutes before induction of UTI. UTI was induced by intravesical inoculation of $4 \times 10^{8} \mathrm{CFU}$ of the uropathogenic Escherichia coli (UPEC type CFT073, ATCC, USA) in $20 \mu \mathrm{L}$ PBS. Liquid collodion (MMD0014, Mavidon, At Erica, The Netherlands) was placed in the urethra for 3 hours for urethral obstruction and mice were sacrificed 36 hours after infection. Bladders and kidneys were removed and fixed in $10 \%$ buffered formalin and embedded in paraffin as previously described [14]. Periodic-acid Schiff stained sections were analysed for the number of renal microabscesses and to score inflammation in the urinary bladder as follows: $0=$ no submucosal edema and neutrophilic infiltrates, 1 = little, 2 = moderate, 3 = strong submucosal edema and neutrophilic infiltrates. Histopathological evaluation was performed by an observer blinded to the treatment conditions. 
Neutrophil counts were assessed from $5 \mu \mathrm{L}$ fresh uncentrifuged urine in a modified Neubauer chamber.

\subsection{Murine bleomycin model}

8- 10 weeks old male C57BL/6J mice obtained from Centro de Bioterismo (CEBIO) of the Universidade Federal de Minas Gerais (UFMG, Brazil) and maintained in the animal facilities of Laboratório de Imunofarmacologia, Department of Biochemistry and Immunology (UFMG, Brazil) were used. Group size was determined with 8 mice each. Animals were anesthetised intraperitoneally with $80 \mu \mathrm{L}$ of a ketamine $\left(3.2 \mathrm{mg} / \mathrm{kg}\right.$, Dopalen ${ }^{\circledR}$, Vetbrands) and xylazine solution $\left(0.16 \mathrm{mg} / \mathrm{kg}\right.$, Calmiun ${ }^{\circledR}$, Agener, União) prior to tracheotomy. A single $25 \mu \mathrm{L}$ injection containing $0.125 \mathrm{U}$ of bleomycin (Blenoxane, Bristol-Meyers) diluted in PBS or PBS only (control group) was instilled intratracheally (i.t.) using Hamilton syringes. 48 hours after bleomycin or PBS instillation, animals were euthanised by a lethal dose of ketamine and xylazine, in order to perform bronchoalveolar lavage (BAL).

Bleomycin-instilled mice were treated twice a day (BID) subcutaneously (s.c.) with vehicle or PA401 (0.01, 0.1 and $1 \mu \mathrm{g} / \mathrm{animal})$ diluted in $100 \mu \mathrm{L}$ of PBS. PA401 was given 3 hours after the bleomycin injection and then administrated twice a day for the following two days. For BAL, the trachea was exposed and a polyethylene catheter was inserted [15]. BAL was performed by washing the lungs three times with $2 \mathrm{~mL}$ PBS each. BAL samples (approximately $1.8-2.0 \mathrm{~mL}$ each) including infiltrated cells were centrifuged at $600 \mathrm{~g}$ for $10 \mathrm{~min}$ at $4{ }^{\circ} \mathrm{C}$. The total number of leukocytes was determined by counting leukocytes in a modified Neubauer chamber. Differential counts were obtained from cytospin (Shandon III, Thermo Scientific, Wilmington, USA) 
preparations by evaluating the percentage of each leukocyte population on a slide stained with May-Grünwald-Giemsa.

For the assessment of CXCL1/KC concentrations in murine lungs ELISA assays were performed using chemokine specific ELISA kits (Mouse CXCL1/KC DuoSet, R\&D Systems, Minneapolis) according to the manufacturer's instruction. Therefore, $100 \mathrm{mg}$ of lung tissue were homogenised in $1 \mathrm{~mL}$ PBS containing anti-proteases $(0.1 \mathrm{mM}$ phenylmethylsulfonyl fluoride, $0.1 \mathrm{mM}$ benzethonium chloride, $10 \mathrm{mM}$ EDTA, $20 \mathrm{KI}$ Aprotinin A) and $0.05 \%$ Tween 20. The samples were then centrifuged for $10 \mathrm{~min}$ at $3000 \mathrm{~g}$ and the supernatant immediately used for ELISA. All samples were assayed in duplicates.

\subsection{Experimental autoimmune uveitis (EAU) in rats}

Uveitis was induced in Lewis rats at the age of 6-8 weeks (Janvier, Le-Genest-St-Isle, France). Rats were maintained under SPF conditions. Treatment of animals was approved by the local government and confirmed to the Association for Research in Vision and Ophthalmology Statement on the use of Animals in Ophthalmic and Vision Research.

The uveitogenic peptide PDSAg was derived from the sequence of bovine retinal S-Antigen (UniProt ID \#P08168, amino acids 342-355, FLGELTSSEVATEV) and obtained from Biotrend (Cologne, Germany).

Rats were subcutaneously immunised into both hind legs with $15 \mu \mathrm{g}$ of peptide in complete Freund's adjuvant, fortified with Mycobacterium tuberculosis strain H37RA (BD, Heidelberg, Germany) to a final concentration of $2.5 \mathrm{mg} / \mathrm{mL}$ as described [16]. For the treatment with PA401, 
groups of 4 rats received daily intraperitoneal injections of $100 \mu \mathrm{g} / \mathrm{rat}$ PA401 in PBS or PBS only from the day of immunisation (day 1) until day 8 (pre-symptomatic treatment) or from day 9 until day 16 (symptomatic treatment). The time course of disease was determined by daily examination of animals with an ophthalmoscope until termination of the experiment at day 20. Uveitis was graded clinically as described by de Smet et al. [17]: score 0, no signs of inflammation; 0.5 , dilated

iris vessels; 1 , peripupillar infiltrates; 2, pupil covered with fibrin; 3, hypopyon and 4, hypopyon with haemorrhage. The score is displayed as the average clinical score of all eyes per group and day. The mean area under the curve defined the average intensity of uveitis in each eye in a group over a certain period of time and was calculated as the summation of the daily clinical uveitis scores for each eye of each rat in a group. For histological analysis eyes from sacrificed animals were immediately snap frozen in methyl butane. Cryosections of rat eyes were stained with hematoxilin and graded for retinal infiltration and destruction as previously described [17].

\subsection{Statistical Analysis}

Data were expressed as mean \pm SEM. Groups were compared using Student`s t-test (ELICO, UTI model), one way ANOVA (Transwell) and ANOVA followed by a Dunnett test (Bleomycin) using GraphPad Prism ${ }^{\circledR}$ version 5.0 (Graphpad Software). Statistics for EAU was performed using InStat 3 software (GraphPad, La Jolla, CA, USA). Nonparametric ANOVA (Kruskal-Wallis test followed by Dunn's multiple comparison test) was used to determine significance levels. A value of $\mathrm{p}<0.05$ was considered to indicate statistical significance. 


\section{RESULTS}

\subsection{Transendothelial migration assay}

We have investigated the inhibitory activity of PA401 on CXCL8-induced neutrophil transendothelial migration in a fully humanised transwell chemotaxis set-up (see Figure 1). The inhibition of transmigration by PA401 was found to be most effective at a concentration of 10 $\mu \mathrm{g} / \mathrm{mL}$ which reduced the CXCL8-induced neutrophil migration by $75 \%$. In the same set-up we investigated the major GAG classes (using the same inhibitory concentration as for PA401) for their transmigration inhibitory activity as comparators to PA401. Interestingly, under these conditions CS showed the strongest inhibition (35\%) followed by unfractionated, high-molecular weight heparin (30\%). Assuming that both, PA401 and GAGs, act similarly in the transwell chemotaxis by displacing cell-bound CXCL8 from endogenous GAGs, PA401 seems to be much more effective. This can be due to the fact that PA401 is expected to act only on CXCL8-specific GAG sites, whereas the GAGs we have used in the current experiment could interact unspecifically with many other proteins presented on the cell surface.

\subsection{ELICO- chemokine displacement fingerprint}

To check if PA401 is indeed specific in displacing only (or mainly) CXCL8 from GAGs, we have established the so-called ELICO assay in which biotinylated chemokines were bound onto GAGcoated microwell plates [12]. These chemokines were then displaced by PA401 in a concentrationdependent manner and the IC50 value for each chemokine was calculated. Taken together these results give the so-called displacement fingerprint for PA401. The ultimate biological relevance of this fingerprint is dependent upon the number of CXCL8 unrelated GAG-binding proteins used in this assay. Here we have used 9 chemokines involved in early inflammatory responses. As can 
be seen in Figure 2, PA401 was most actively displacing CXCL8 from HS with an IC50 value of 236 nM. PA401 displaced, however, also CCL2 with a similar IC50 value (261 nm). When wild type CXCL8 was used as a competitor in the fingerprint assay, it was found to displace CXCL8 and CCL2 from HS with comparable IC50 values as well, although with higher IC50 values compared to PA401 (data not shown). It may therefore be assumed that the GAG pattern recognised by these two chemokines is overall very similar. If a GAG (heparin, HS or CS) was used as a competitor in the ELICO set-up, much higher IC50 values (i.e. in the $\mu \mathrm{M}$ range) were obtained to displace CXCL8 from HS (data not shown). This is a further indication for the low specificity of generic GAGs as competitors of protein-GAG interactions.

\subsection{PA401 in urinary tract infection}

In a first in vivo assessment of PA401's ability to inhibit neutrophil migration, $1 \mu \mathrm{g}$ of the compound was given i.p. 10 min before the induction of UTI by the uropathogenic E. coli strain. The initial dose ( $1 \mu \mathrm{g}$ PA401 per mouse $=130$ pmol per animal $)$ was chosen in order to be above the IC50 value found for PA401 in the ELICO experiment with respect to CXCL8 displacement. An evaluation of migrated neutrophils assessed from $5 \mu \mathrm{L}$ fresh non-centrifuged urine in the PA401 group showed a significant decrease of migrated cells compared to the control treatment (see Figure 3A) indicating a significant inhibition of inflammatory cells recruitment. Furthermore, a strong PA401-related reduction of inflammation was also observed in histological sections of renal micro-abscesses (shown in Figure 3B), in which typical inflammatory infiltrates were detected in UTI-induced, vehicle (saline)-treated mice, in comparison to the normalisation of the tissue architecture observed in PA401-treated mice. In addition, the number of abscesses per kidney section was also reduced in the PA401 treated group (data not shown). Taken together, 
mice treated with PA401 showed a strongly mitigated form of inflammation which was expressed in the three typical pathological parameters measured in this study.

\subsection{PA401 in bleomycin-induced lung inflammation}

In order to test the inhibitory efficacy of PA401 on neutrophil migration in a different organ, bleomycin was used as a trigger for recruitment of cells into lungs. BAL was analysed with regard to the total number of recruited cells and to the number of recruited neutrophils (see Figure 4A). Bleomycin injected i.t. induced strong and reproducible cell mobilisation into murine lungs at 48 h post injection. When PA401 was administrated s.c., a significant reduction in total cell counts and neutrophils was observed at $0.1 \mu \mathrm{g}$ and $1 \mu \mathrm{g} /$ mouse, the highest effect being observed at $1 \mu \mathrm{g}$ PA401 (see Figure 4A).

KC (murine CXCL1) is - amongst others - typically released during the early inflammatory phases and is a pro-inflammatory cytokine responsible for neutrophil recruitment. KC was, indeed, found in murine lungs at high concentrations after bleomycin instillation (average $760 \mathrm{ng} / 100 \mathrm{mg}$ of lung tissue analysed by ELISA). Treatment with PA401 unexpectedly decreased the amount of KC significantly already at the lowest PA401 dose applied (0.01 $\mu$ g) (see Figure 4B).

\subsection{PA401 in experimental autoimmune uveitis}

EAU uveitis is characterised by massive infiltration of inflammatory cells like neutrophils and macrophages in the eyes. In order to investigate not only the well-known involvement of CXCL8 in this disease but also the participation of GAGs in disease onset and progression, the effect of PA401 on clinical uveitis scores (anterior chamber inflammation) and destruction of the retina 
(histological evaluation) after either pre-symptomatic or symptomatic treatment regimen was evaluated. Active immunisation with PDSAg resulted in the development of autoimmune uveitis in the vehicle treated group of rats from day 9 post immunisation. PA401 i.p. daily treatment at the dose of $100 \mu \mathrm{g} / \mathrm{rat}$ under both pre-symptomatic and symptomatic treatment regimen was able to modify disease progression with respect to severity and incidence of disease (Fig. 5). The clinical course of EAU was significantly ameliorated during peak disease in the group treated with PA401 from onset of clinical signs (PA401 day 9-16) and strongly reduced in rats treated from immunisation until the day before onset of uveitis (Fig. 5A, B).

The mean maximal clinical disease scores were reduced in both treatment groups and a slight attenuating effect on the retinal destruction was also observed by histology after PA401 treatment (Fig. 5C, D). In the PBS-treated group, all rats reached uveitis score 3, while in the treated groups a few eyes had weak or even no disease, 3 eyes in the group treated from day 1-8 and one eye in the group treated after onset from day 9-16 were devoid of any signs of uveitis. In the symptomatically treated group the treatment was stopped at day 16 , when the clinical signs of uveitis had almost resolved. Surprisingly, in one rat both eyes showed inflammation (clinical scores 2) the next day, and in two other rats the inflammation increased again in one and two eyes, respectively, indicating the control of inflammation by PA401 even when treatment was initiated only after onset of inflammation. In the group treated from immunisation to the day just before clinical onset of uveitis we also observed a strong suppression of inflammation, but 8 days after cessation of PA401 injection two previously unaffected eyes in two rats suddenly developed uveitis (Fig. 5A). 


\section{DISCUSSION}

We have engineered human CXCL8 with respect to its binding to GAGs (high affinity) and to CXCR1/2 (knocked-out) [10]. This decoy protein PA401 was found to be active in several animal models of inflammatory diseases $[18,19]$. Here we used this chemokine mutant to investigate its molecular mode of action as inhibitor of neutrophil migration and to thereby clearly relate to the influence of GAGs in CXCL8-mediated inflammation. For other chemokines, namely MCP1/CCL2, RANTES/CCL5 and MIP-1ß/CCL4, the importance of GAG-binding in leukocyte migration was shown in murine peritonitis by knocking-out their GAG-binding domain [20, 21]. For CXCL8, however, this was never shown clearly. Moreover, in this study we had a closer look at the selectivity and specificity of the chemokine-GAG interaction.

CXCL8 is the prototypic neutrophil-attracting/activating chemokine. The high-affinity protein receptors on leukocytes have been identified to be CXCR1 and CXCR2 [22, 23], whereas as proteoglycan co-receptors syndecan-1 to syndecan-4 have been proposed [11, 24-26]. In addition, a GAG oligosaccharide sequence has been suggested by Lindahl and co-workers to be the specific GAG epitope for CXCL8 binding [27]. If thus the dominant-negative CXCL8 isoform described here exhibits anti-migratory and anti-inflammatory effects in vitro and in vivo, this can only be explained by the enhanced GAG binding affinity of the mutant PA401 which leads to displacement

of the wild type chemokine from its cognate GAG receptor. Consequently, CXCL8-mediated inflammation, and particularly chemotaxis, is dependent upon the involvement of GAGs in these processes. 
From the transwell experiments described here, it became clear that CS and high molecular weight heparin were primarily able to prevent neutrophils from transendothelial migration. These results relate to the fact that CXCL8 binds preferably to these two GAG classes. However, for effectively competing CXCL8 from endothelial cells, i.e. to inhibit transendothelial migration, relatively high concentrations are required (see Figure 1). This could explain the frequently observed mild and unspecific anti-inflammatory effects of heparins during anti-thrombotic treatment of human subjects [28, 29]. At the concentration of $10 \mu \mathrm{g} / \mathrm{mL}$ PA401 inhibited neutrophil transmigration to a much higher extent, highlighting its potent anti-migratory properties. In our structure-based engineering approach we aimed at maintaining the specificity of the wild type chemokine for its respective GAG co-receptor, allowing for a specific and effective displacement of CXCL8.

By using the ELICO fingerprint assay, in which we mimic the glycocalyx by coating a plate with GAGs, we were able to analyse the displacement pattern of PA401. In this assay we could show that different chemokines are displaced to a different extent by PA401, confirming that there is a certain level of specificity in the chemokine-GAG interaction. Furthermore, we want to highlight the importance of considering the displacement profile of chemokine mutants or GAG mimetics designed for therapeutic purposes.

These findings were then complemented with three in vivo models of neutrophil-mediated inflammation.

Urinary tract infection (UTI) occurs in otherwise healthy persons, in case fecal bacteria accidentally access the urinary tract through the urethra and then colonise the bladder inducing inflammation and symptoms clinically characterised as cystitis. If untreated, the bacteria can 
ascend the ureters and colonise the kidneys, resulting in a more serious secondary infection at this level. A number of secreted factors such as cytokines and chemokines are detected in the mammalian bladder upon infection and are leading to the recruitment of cellular infiltrates, among which the neutrophils are the most abundant early responders [30]. Elevated urinary CXCL8 levels have been reported in patients affected by UTI [31], and the GAG layer is known to be damaged in chronic UTI, however the role played by GAGs in the presentation of CXCL8 and therefore in neutrophils recruitment in the early UTI phase has been unknown. We have therefore tested the ability of PA401 to inhibit neutrophil recruitment in a murine model of UTI, evaluating neutrophil infiltration both in the lower urinary tract, by measuring neutrophils in the urine, as well as by assessing at histological level bladder inflammation and renal microabscess. The results suggest that displacement of CXCL8 by GAGs, by means of PA401 treatment, reduced neutrophil recruitment and bladder inflammation, without affecting the ability to block the pathogen ascension to the kidneys, as demonstrated by some normalisation of kidney histology.

We then explored the effects on PA401 in a murine model of pulmonary fibrosis, which is characterised by chronic inflammation followed by extensive collagen deposition resulting, on long term, in loss of lung functions. Bleomycin single intra-tracheal instillation in the lung is the most frequently used experimental preclinical model to investigate early cellular and biochemical mechanisms relevant for the pathogenesis of pulmonary fibrosis. The relevance of this animal model for human pathology is further supported by the knowledge that bleomycin's potent anticancer therapeutic efficacy in humans is limited by the risk of development of lung fibrosis to it associated [32]. 
The pulmonary response to bleomycin injury is complex and involves the coordinated expression and secretion of various cytokines and chemokines at the site of injury, driving the migration of leukocytes, which are primarily neutrophils and monocytes in the first couple of days [33].

PA401 was able to reduce dose-dependently the recruitment of inflammatory cells into the lung, as measured by cell count in the bronchoalveolar lavage (BAL) samples, and with strong activity on neutrophils, even when administered at a dose as low as $0.1 \mu \mathrm{g} /$ mouse s.c. The reduction in inflammatory cell infiltrate was associated to a significant reduction in KC levels in lung homogenate samples.

This result indicates that $\mathrm{KC}$ was quickly removed or degraded from lungs once it became displaced by PA401, and is in line with what we have already reported for PA401 in other murine models of lung inflammation [11, 19], as well as for PA401 in vitro activity on CXCL8 in BAL samples from cystic fibrosis patient [18]. Mechanistically, GAGs seem to be therefore involved in bleomycin-induced leukocyte recruitment into the lungs by presenting at least $\mathrm{KC}$, the murine CXCL8 functional analogue, to migrating cells.

Experimental autoimmune uveitis is a CD4+ T cell mediated disease with intraocular inflammation and destruction of the retina caused by inflammatory cells (monocytes/macrophages and neutrophil granulocytes) recruited by autoreactive T cells, which infiltrated the eyes and secrete cytokines and chemokines to recruit the inflammatory cells from the circulation. We have previously shown that the autoreactive $\mathrm{T}$ cells have infiltrated the eyes already three days before any clinical signs of inflammation are visible [34]. Thus, even cessation of treatment before onset of clinical disease could have an impact by affecting the early stages of recruitment of 
inflammatory cells. The immigration of inflammatory cells can destroy the blood-retina barrier and thus enable even more cells to easily invade the eyes from the circulation; therefore early prevention of inflammation is an important therapeutic goal. The late onset in some animals 8 days after termination of the preventive therapy might be explained by the fact that we still have antigen at the site of immunisation and therefore autoreactive $\mathrm{T}$ cells can be activated later, when the therapeutic drug is not applied any more. This suggests that the inhibition of CXCL8 could also play a pivotal role in the activation of autoreactive $\mathrm{T}$ cells and not only in the recruitment of granulocytes, since CXCL8 together with IL-6 and VEGF has been shown to induce a dendritic cell subset that can suppress $\mathrm{T}$ cell responses [35]. The strong and fast rebound effect immediately after cessation of the symptomatic therapy might be rather an effect on inflammatory cell recruitment than on $\mathrm{T}$ cell activation. Taken together, although rodents do not have CXCL8, the effect of human CXCL8 on rat immune cells has been shown before [36] and is further demonstrated by the effect of PA401 in this rat model of human autoimmune uveitis. In addition, by the dominant-negative nature of PA401, the anti-inflammatory activity of this decoy clearly points out the strong involvement of GAGs in the progress of this disease.

\section{CONCLUSION}

In this study, we have confirmed the pivotal role of GAGs in CXCL8-related, neutrophil-mediated inflammatory diseases which further confirms that the interaction between GAGs and chemokines can be exploited therapeutically. Furthermore, we were able to show that there is a certain degree of selectivity (interaction preferably with a distinct type of GAG) and specificity (interaction with a particular domain/sequence within a certain GAG type) in this interaction. 


\section{FUNDING}

Martha Gschwandtner was recipient of a DOC Fellowship of the Austrian Academy of Sciences at the Institute of Pharmaceutical Chemistry, University of Graz, Austria. Elisabeth Strutzmann was supported by the FFG (Österreichische Forschungsförderungsgesellschaft).

\section{CONFLICT OF INTEREST}

The authors declare that there is no conflict of interest. 


\section{REFERENCES}

[1] N. Mukaida, Pathophysiological roles of interleukin-8/CXCL8 in pulmonary diseases, Am. J. Physiol. Lung Cell Mol. Physiol., 284 (2003) L566-577.

[2] D. D'Ambrosio, P. Panina-Bordignon, F. Sinigaglia, Chemokine receptors in inflammation: an overview, J. Immunol. Methods, 273 (2003) 3-13.

[3] T.L. Bonfield, J.R. Panuska, M.W. Konstan, K.A. Hilliard, J.B. Hilliard, H. Ghnaim, M. Berger, Inflammatory cytokines in cystic fibrosis lungs, Am. J. Respir. Crit. Care Med., 152 (1995) 2111-2118.

[4] G.S. Kuschert, F. Coulin, C.A. Power, A.E. Proudfoot, R.E. Hubbard, A.J. Hoogewerf, T.N. Wells, Glycosaminoglycans interact selectively with chemokines and modulate receptor binding and cellular responses, Biochemistry, 38 (1999) 12959-12968.

[5] N. Afratis, C. Gialeli, D. Nikitovic, T. Tsegenidis, E. Karousou, A.D. Theocharis, M.S. Pavão, G.N. Tzanakakis, N.K. Karamanos, Glycosaminoglycans: key players in cancer cell biology and treatment, FEBS J, 279 (2012) 1177-1197.

[6] A.E.I. Proudfoot, The biological relevance of chemokine-proteoglycan interactions, Biochem. Soc. Trans., 34 (2006) 422-426.

[7] D. Xu, J.D. Esko, Demystifying heparan sulfate-protein interactions, Annu. Rev. Biochem., 83 (2014) 129-157.

[8] G.S. Kuschert, A.J. Hoogewerf, A.E. Proudfoot, C.W. Chung, R.M. Cooke, R.E. Hubbard, T.N. Wells, P.N. Sanderson, Identification of a glycosaminoglycan binding surface on human interleukin-8, Biochemistry, 37 (1998) 11193-11201.

[9] G. Williams, N. Borkakoti, G.A. Bottomley, I. Cowan, A.G. Fallowfield, P.S. Jones, S.J. Kirtland, G.J. Price, L. Price, Mutagenesis studies of interleukin-8. Identification of a second epitope involved in receptor binding, J. Biol. Chem., 271 (1996) 9579-9586.

[10] A. Falsone, V. Wabitsch, E. Geretti, H. Potzinger, T. Gerlza, J. Robinson, T. Adage, M.M. Teixeira, A.J. Kungl, Designing CXCL8-based decoy proteins with strong anti-inflammatory activity in vivo, Biosci. Rep., 33 (2013).

[11] T. Adage, V. Konya, C. Weber, E. Strutzmann, T. Fuchs, C. Zankl, T. Gerlza, D. Jeremic, A. Heinemann, A.J. Kungl, Targeting glycosaminoglycans in the lung by an engineered CXCL8 as a novel therapeutic approach to lung inflammation, Eur. J. Pharmacol., 748 (2015) 83-92. [12] T. Gerlza, B. Hecher, D. Jeremic, T. Fuchs, M. Gschwandtner, A. Falsone, B. Gesslbauer, A.J. Kungl, A combinatorial approach to biophysically characterise chemokine-glycan binding affinities for drug development, Molecules, 19 (2014) 10618-10634.

[13] M. Gschwandtner, A.M. Piccinini, T. Gerlza, T. Adage, A.J. Kungl, Interfering with the CCL2-glycosaminoglycan axis as a potential approach to modulate neuroinflammation, Neurosci. Lett., 626 (2016) 164-173.

[14] P.S. Patole, S. Schubert, K. Hildinger, S. Khandoga, A. Khandoga, S. Segerer, A. Henger, M. Kretzler, M. Werner, F. Krombach, D. Schlöndorff, H.-J. Anders, Toll-like receptor-4: renal cells and bone marrow cells signal for neutrophil recruitment during pyelonephritis, Kidney Int., 68 (2005) 2582-2587.

[15] R.C. Russo, R. Guabiraba, C.C. Garcia, L.S. Barcelos, E. Roffê, A.L.S. Souza, F.A. Amaral, D. Cisalpino, G.D. Cassali, A. Doni, R. Bertini, M.M. Teixeira, Role of the chemokine receptor CXCR2 in bleomycin-induced pulmonary inflammation and fibrosis, Am. J. Respir. Cell Mol. Biol., 40 (2009) 410-421. 
[16] M. Diedrichs-Möhring, P.J. Nelson, A.E.I. Proudfoot, S.R. Thurau, G. Wildner, The effect of the CC chemokine receptor antagonist Met-RANTES on experimental autoimmune uveitis and oral tolerance, J. Neuroimmunol., 164 (2005) 22-30.

[17] M.D. de Smet, G. Bitar, F.G. Roberge, I. Gery, R.B. Nussenblatt, Human S-antigen:

presence of multiple immunogenic and immunopathogenic sites in the Lewis rat, J. Autoimmun., 6 (1993) 587-599.

[18] O.J. McElvaney, N. O'Reilly, M. White, N. Lacey, K. Pohl, T. Gerlza, D.A. Bergin, H. Kerr, C. McCarthy, M.E. O'Brien, T. Adage, A.J. Kungl, E.P. Reeves, N.G. McElvaney, The effect of the decoy molecule PA401 on CXCL8 levels in bronchoalveolar lavage fluid of patients with cystic fibrosis, Mol. Immunol., 63 (2015) 550-558.

[19] T. Adage, F. Del Bene, F. Fiorentini, R.P. Doornbos, C. Zankl, M.R. Bartley, A.J. Kungl, PA401, a novel CXCL8-based biologic therapeutic with increased glycosaminoglycan binding, reduces bronchoalveolar lavage neutrophils and systemic inflammatory markers in a murine model of LPS-induced lung inflammation, Cytokine, 76 (2015) 433-441.

[20] E.K. Lau, C.D. Paavola, Z. Johnson, J.-P. Gaudry, E. Geretti, F. Borlat, A.J. Kungl, A.E. Proudfoot, T.M. Handel, Identification of the glycosaminoglycan binding site of the CC chemokine, MCP-1: implications for structure and function in vivo, J. Biol. Chem., 279 (2004) 22294-22305.

[21] A.E.I. Proudfoot, T.M. Handel, Z. Johnson, E.K. Lau, P. LiWang, I. Clark-Lewis, F. Borlat, T.N.C. Wells, M.H. Kosco-Vilbois, Glycosaminoglycan binding and oligomerization are essential for the in vivo activity of certain chemokines, Proc. Natl. Acad. Sci. U. S. A., 100 (2003) 1885-1890.

[22] P.M. Murphy, H.L. Tiffany, Cloning of complementary DNA encoding a functional human interleukin-8 receptor, Science, 253 (1991) 1280-1283.

[23] W.D. Holmes, T.G. Consler, W.S. Dallas, W.J. Rocque, D.H. Willard, Solution studies of recombinant human stromal-cell-derived factor-1, Protein Expr. Purif., 21 (2001) 367-377.

[24] A.M. Patterson, L. Gardner, J. Shaw, G. David, E. Loreau, L. Aguilar, B.A. Ashton, J. Middleton, Induction of a CXCL8 binding site on endothelial syndecan-3 in rheumatoid synovium, Arthritis Rheum., 52 (2005) 2331-2342.

[25] Y. Halden, A. Rek, W. Atzenhofer, L. Szilak, A. Wabnig, A.J. Kungl, Interleukin-8 binds to syndecan-2 on human endothelial cells, Biochem. J., 377 (2004) 533-538.

[26] M. Gotte, F. Echtermeyer, Syndecan-1 as a regulator of chemokine function, ScientificWorldJournal, 3 (2003) 1327-1331.

[27] D. Spillmann, D. Witt, U. Lindahl, Defining the interleukin-8-binding domain of heparan sulfate, J. Biol. Chem., 273 (1998) 15487-15493.

[28] L.J. Downing, R.M. Strieter, A.M. Kadell, C.A. Wilke, L.J. Greenfield, T.W. Wakefield, Low-dose low-molecular-weight heparin is anti-inflammatory during venous thrombosis, $\mathrm{J}$. Vasc. Surg., 28 (1998) 848-854.

[29] D.J. Tyrrell, A.P. Horne, K.R. Holme, J.M. Preuss, C.P. Page, Heparin in inflammation: potential therapeutic applications beyond anticoagulation, Adv. Pharmacol., 46 (1999) 151-208. [30] M. Haraoka, L. Hang, B. Frendéus, G. Godaly, M. Burdick, R. Strieter, C. Svanborg, Neutrophil recruitment and resistance to urinary tract infection, J. Infect. Dis., 180 (1999) 12201229.

[31] Y.C. Ko, N. Mukaida, S. Ishiyama, A. Tokue, T. Kawai, K. Matsushima, T. Kasahara, Elevated interleukin-8 levels in the urine of patients with urinary tract infections, Infect. Immun., 61 (1993) 1307-1314. 
[32] S. Sleijfer, Bleomycin-induced pneumonitis, CHEST Journal, 120 (2001) 617-624.

[33] N.I. Chaudhary, A. Schnapp, J.E. Park, Pharmacologic differentiation of inflammation and fibrosis in the rat bleomycin model, Am. J. Respir. Crit. Care Med., 173 (2006) 769-776.

[34] S.R. Thurau, T.R. Mempel, A. Flügel, M. Diedrichs-Möhring, F. Krombach, N. Kawakami, G. Wildner, The fate of autoreactive, GFP+ T cells in rat models of uveitis analyzed by intravital fluorescence microscopy and FACS, Int. Immunol., 16 (2004) 1573-1582.

[35] A.-M. Figel, D. Brech, P.U. Prinz, U.K. Lettenmeyer, J. Eckl, A. Turqueti-Neves, J.

Mysliwietz, D. Anz, N. Rieth, N. Muenchmeier, Human renal cell carcinoma induces a dendritic cell subset that uses T-cell crosstalk for tumor-permissive milieu alterations, The American journal of pathology, 179 (2011) 436-451.

[36] A. Rot, Chemotactic potency of recombinant human neutrophil attractant/activation protein1 (interleukin-8) for polymorphonuclear leukocytes of different species, Cytokine, 3 (1991) 2127. 


\section{FIGURES AND LEGENDS}

Figure 1: Transendothelial migration assay. Inhibitory setup using $10 \mathrm{nM}$ CXCL8 as

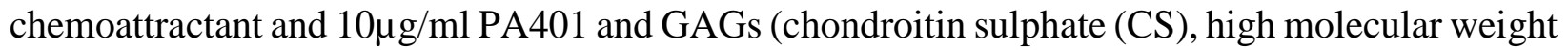
heparin (HMWH), low molecular weight heparin (LMWH), dermatan sulphate (DS), heparan sulphate (HS)) as inhibitors of transmigration, EA.hy926 monolayer and human neutrophils, mean \pm SEM. ${ }^{*} \mathrm{p}<0.05, * * * \mathrm{p}<0.001$.

Figure 2: ELICO-based fingerprint. PA401 displacement profile of various chemokines from immobilised heparan sulphate (HS). ${ }^{* *} \mathrm{p}<0.01,{ }^{* * * *} \mathrm{p}<0.0001$ versus CXCL8.

Figure 3: E. coli-induced urinary tract infection. (A) Urine neutrophils, mean $\pm S E M,{ }^{*} p<0.05$. (B) Kidney periodic-acid Schiff stained histopathology sections (400x magnification). Photomicrograph illustrates infiltration of granulocytes indicating acute inflammation.

Figure 4: Bleomycin-induced lung inflammation. (A) Left, number of total cell and (right) neutrophils detected in the BAL using Neubauer cell chamber and cytospin analysis, mean \pm SEM. (B) amount of detectable CXCL1 (KC) in lung homogenate. ${ }^{*} \mathrm{p}<0.05,{ }^{* *} \mathrm{p}<0.01,{ }^{* * *} \mathrm{p}<0.001$.

Figure 5: PDSAg-induced experimental autoimmune uveitis. (A) Course of EAU after presymptomatic (day 0-8) and symptomatic (day 9-16) treatment with PA401. Mean daily clinical scores of all eyes from each group \pm SEM are shown $(n=8)$. The PBS-treated control group was injected daily from immunisation to day 16. (B) Mean area under the curve \pm SEM calculated for each eye in a group from onset (day 9) to the termination of the experiment at day 20. (C) Mean maximal clinical uveitis scores for all eyes of each group (clinical uveitis score) and mean maximal 
histological scores \pm SEM are shown ( $\mathrm{n}=8$ eyes/group). (D) Representative cryosections of retinas are shown for each group (as indicated). 
Figure 1

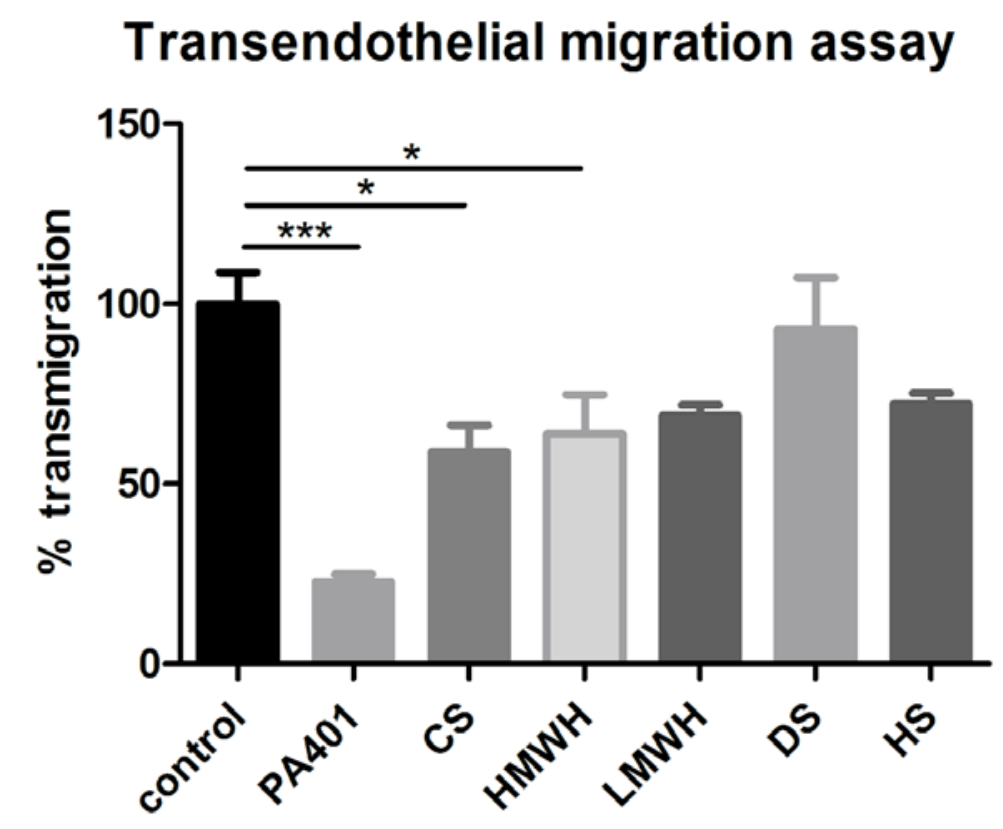

Figure 2

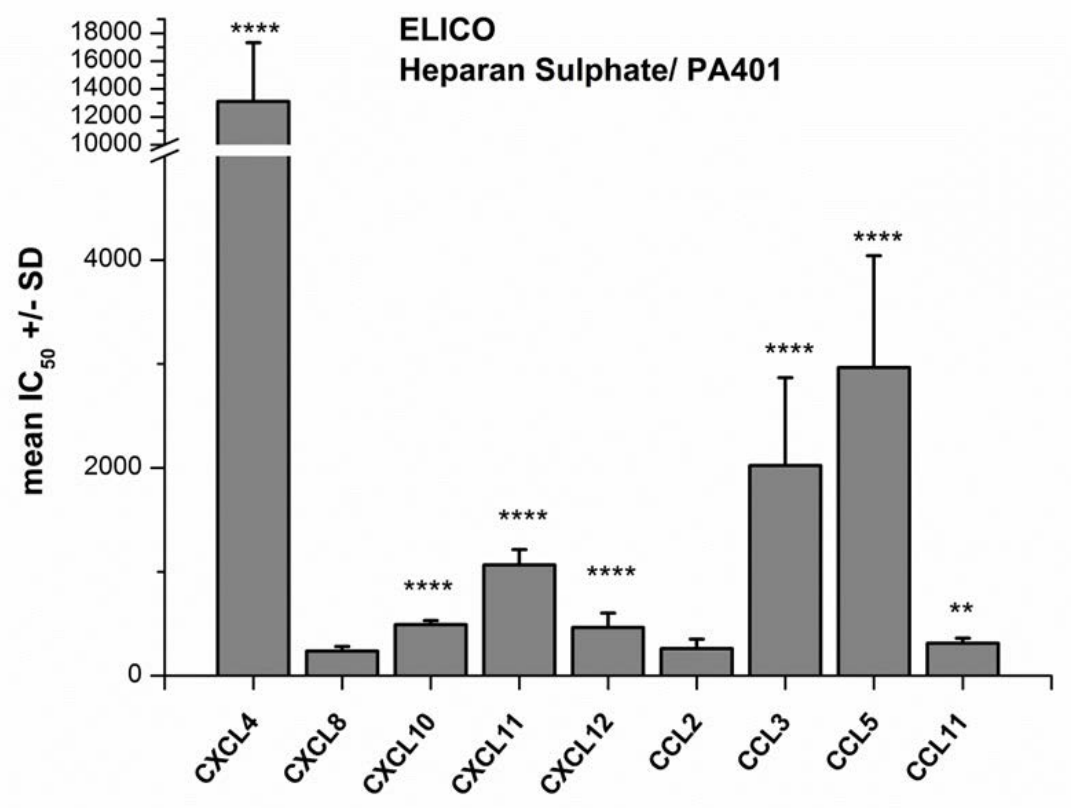


Figure 3A

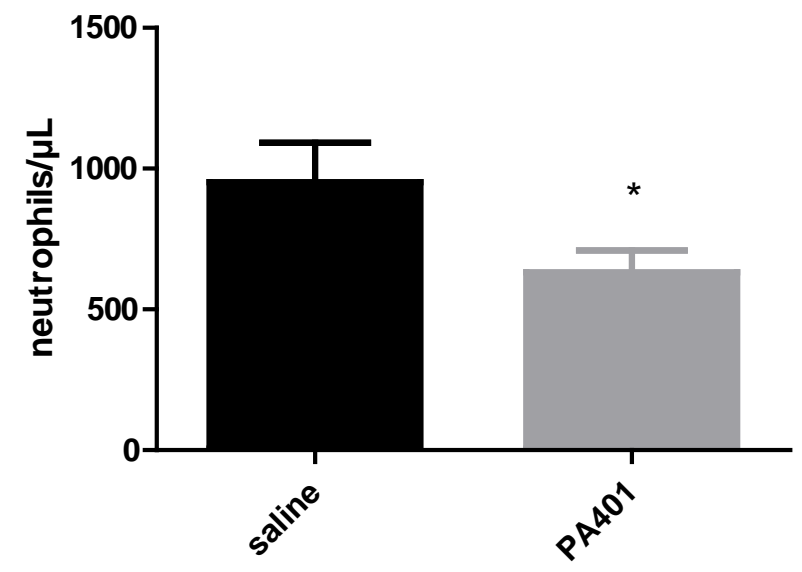

Figure 3B
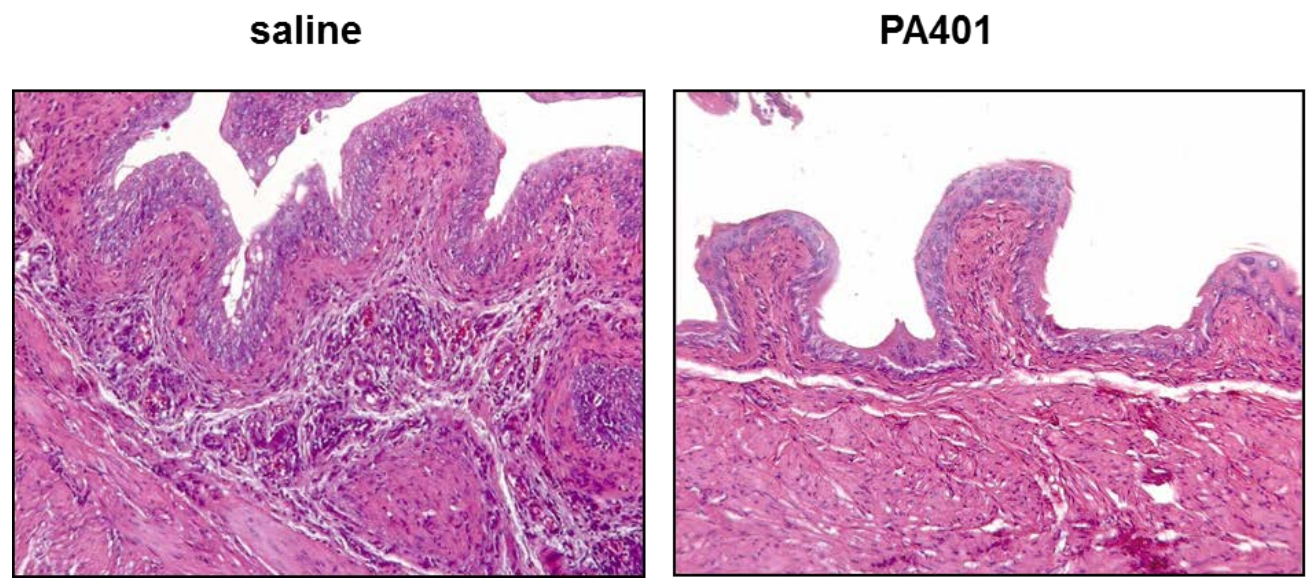
Figure 4A
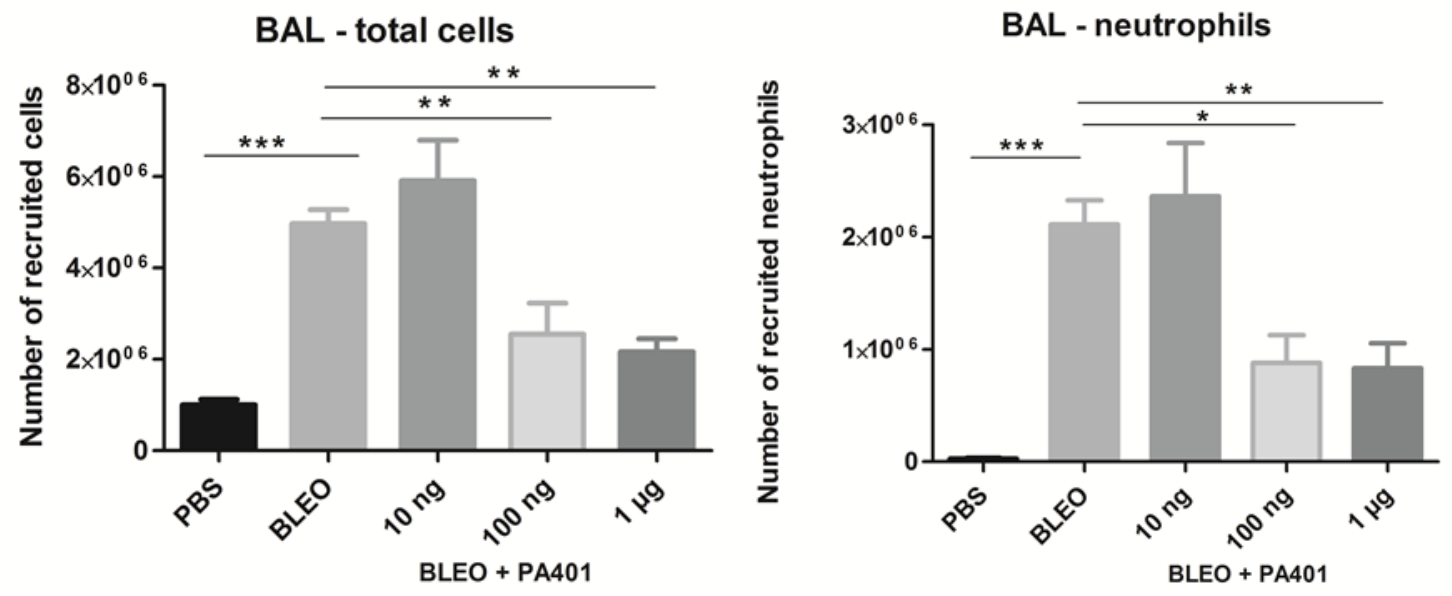

Figure 4B

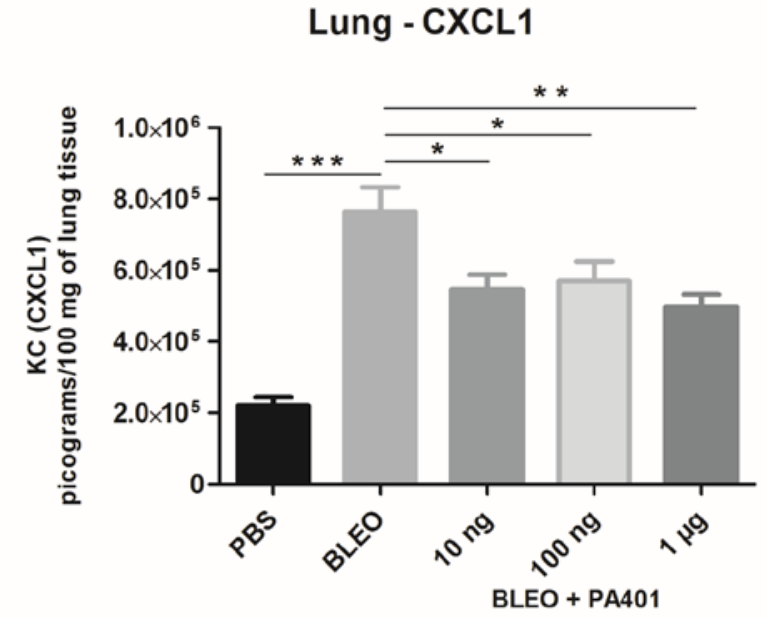


Figure 5A

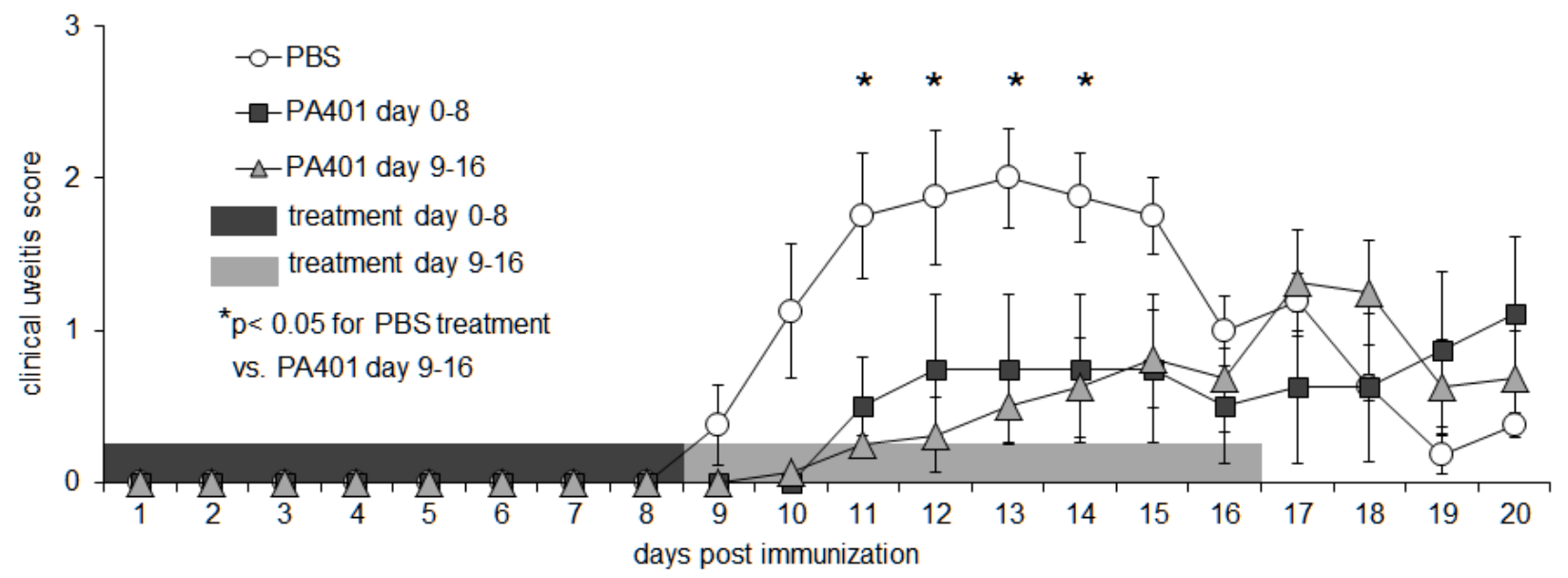

Mean daily uveitis scores \pm SE for each group

\section{Figure 5B}

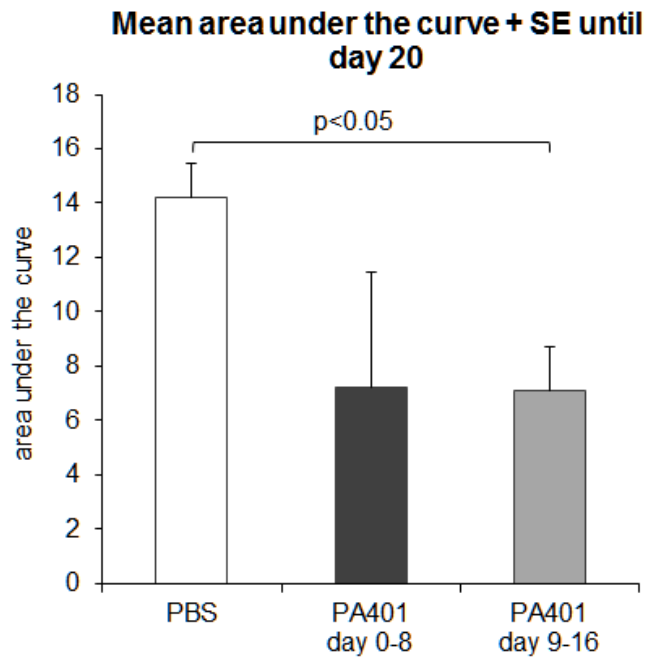


Figure 5C

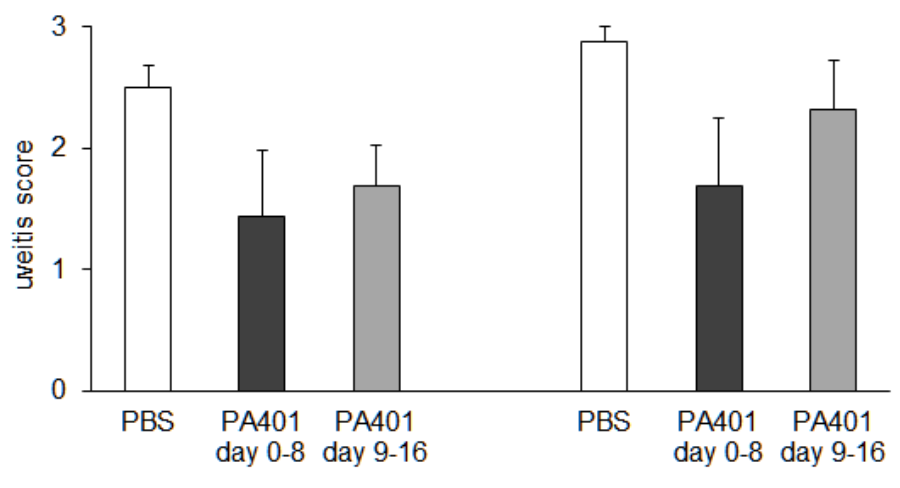

clinical uveitis score

histological uveitis score

\section{Figure 5D}

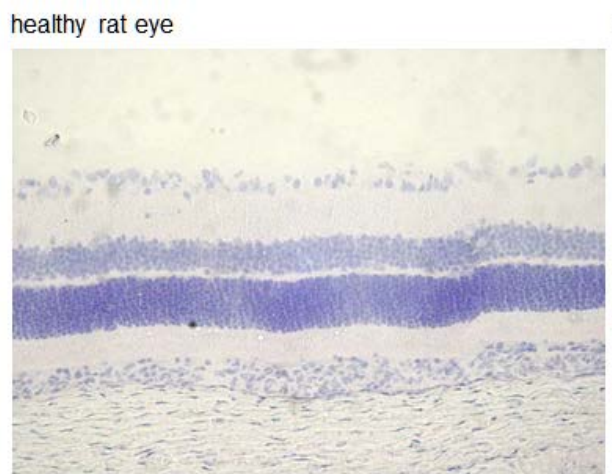

PBS-treated control group

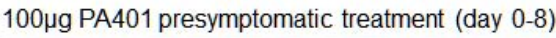
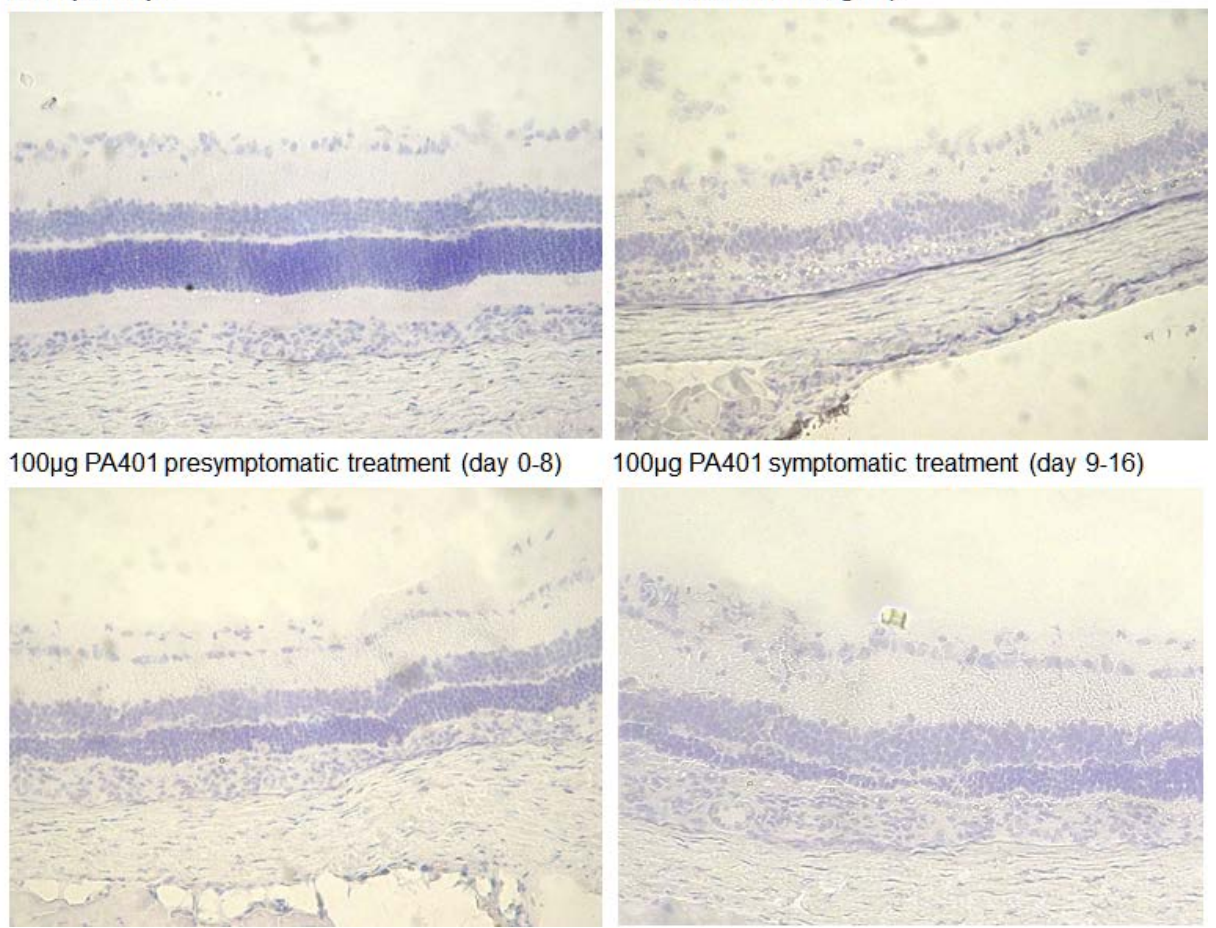

$100 \mu \mathrm{g}$ PA401 symptomatic treatment (day 9-16)

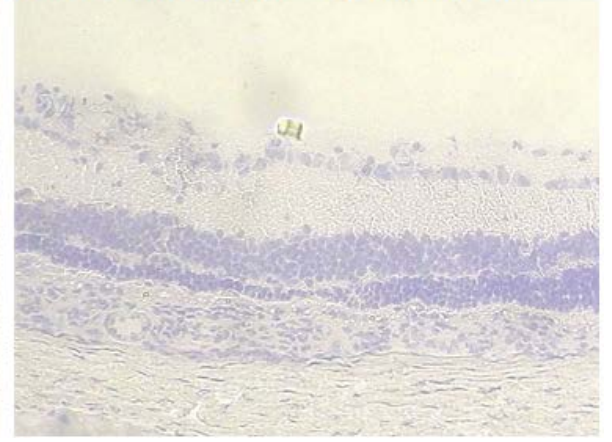

